\title{
Snapshots of proteins at work
}

Two groups extend the boundaries of in-cell nuclear magnetic resonance spectroscopy.

$\mathrm{X}$-ray crystallography and nuclear magnetic resonance (NMR) spectroscopy have been successfully used to determine the threedimensional structures of tens of thousands of proteins. However, these techniques require yanking the protein out of its natural cellular environment and analyzing it in a sterile 'test tube'. Though much can be learned by studying proteins in vitro, researchers would ideally like to have methods to study proteins under physiological conditions.

In-cell NMR spectroscopy is an intriguing technique that is being used to look at proteins at work inside cells. The inside of a cell is a highly crowded space filled with other proteins, lipids, nucleic acids and small molecules, not to mention the confines of the cytoskeleton and various organelles. All of these things can potentially affect protein structure. "Structural analyses of proteins inside cells are interesting because they will tell us whether structures in vitro and in vivo are the same or not," observes Yutaka Ito of Tokyo Metropolitan University.

Ito's group recently explored this question by solving a high-quality structure of the small protein TTHA1718 from Thermus thermophilus using in-cell NMR spectroscopy (Sakakibara et al., 2009). This is the first structure solved exclusively by the incell method without using any structural information from in vitro experiments. To achieve this feat, "since in-cell NMR suffers from low sensitivity, broad overlapping resonance lines and the short lifetime of cell samples, we needed to combine advanced NMR methods, with some further optimizations, to overcome the problems," explains Ito. They improved the spectral quality and reduced the experimental time from 1-2 days to just 2-3 hours needed to analyze the ${ }^{15} \mathrm{~N}$ - and ${ }^{13} \mathrm{C}$-labeled protein, overexpressed in Escherichia coli.

Ito's group found that the in-cell and in vitro structures of TTHA 1718 were very similar. However, they observed some differences at the heavy metal binding site, indicating that interactions with metal ions in the $E$. coli cytosol can affect the protein structure.

Almost all in-cell NMR spectroscopy experiments to date have been performed in E. coli, owing to the challenge of expressing a protein at a high concentration in eukaryotic cells. It is possible to microinject isotopelabeled recombinant proteins into very large cells such as Xenopus oocytes, but Masahiro Shirakawa of Kyoto University sought to develop a more general approach to study proteins in human cells. His group recently harnessed a cell penetrating peptide to deliver ${ }^{15} \mathrm{~N}$-labeled proteins into HeLa cells (Inomata et al., 2009). Notably, as the delivered protein is the only isotope-labeled entity, the spectra are cleaner than those of overexpressed proteins in E. coli, which suffer from high background.

With this method, Shirakawa's group found that ubiquitin in cells was less structured compared to ubiquitin in an in vitro environment. They were surprised by this, because "it has been a general belief that proteins are stabilized in cells," says Shirakawa. "But our results indicate that for ubiquitin it is the opposite." The researchers believe that this may be at least partly due to the interaction of ubiquitin with endogenous proteins.

They also studied the effect of immunosuppressants on the protein FKBP12. "Observations of in-cell NMR using human cells can be directly linked to pharmaceutical [such as drug screening] and medical applications," notes Shirakawa.

Shirakawa also suggests that in-cell NMR spectroscopy could be used to study the aggregation mechanisms of amyloidforming proteins. Ito is particularly interested in applying the technique to intrinsically disordered proteins. Additional methodological developments will be needed to solve structures of larger proteins or of proteins at closeto-endogenous concentrations, but both Ito and Shirakawa believe that many methods developed for in vitro NMR spectroscopy could be directly applied to improve the incell technology.

These developments help further lay the groundwork for an exciting future for in-cell NMR spectroscopy, taking protein structure analysis from the test tube back to the cell.

\section{Allison Doerr}

\section{RESEARCH PAPERS}

Sakakibara, D. et al. Protein structure determination in living cells by in-cell NMR spectroscopy. Nature 458, 102-105 (2009).

Inomata, K. et al. High-resolution multi-dimensional NMR spectroscopy of proteins in human cells. Nature 458, 106-109 (2009). 